\title{
Beiträge zu den älteren und jüngeren germanischen Lehnwörtern im Finnischen
}

\begin{abstract}
A Germanic origin is proposed for the following Finnish words: 1.1. Fi. karhe 'windrow', karhita 'windrow the hay' Germ. *karziz 'turn, wind, change', *karzijan 'turn (round)'; 1.2. Fi. kuono 'muzzle, nose, snout' Germ. *skōnō id.; 1.3. Fi. rakentaa 'build, put up, erect; harness (a horse)' Germ. *rakjan 'put up; stretch'; 1.4. Fi. tana- : tanassa, tanaan 'outstretched, firm, stiff' Germ. *pana 'stretch, tension'. A Swedish origin is proposed for the following Finnish words: 2.1. Fi.dial. ahdike, atturi 'an afternoon meal (snack) in the field during haymaking and harvest time' Old Sw. aptan, aftan, apt- 'evening, afternoon', Sw.dial. afto, aftu 'id.; cold supper, evening meal'; 2.2. Fi.dial. aunehtia 'envy, be envious' Sw.dial. avunna id.; 2.3. Fi.dial. hiiata 'prevent; slow (down, up); dawdle, loiter' Sw.dial. hia id.; 2.4. Fi.dial. hinnerikki 'trouble, hindrance' $\sim$ Sw. hinder id.; 2.5. Fi.dial. illata 'agree, approve' $\sim$ Sw. gilla id.; 2.6. Fi. inttää 'object; argue; deny' Sw. int(e) 'not'; 2.7. Fi.dial. kyönätä 'notice; understand' Sw. skön(j)a id.; 2.8. Fi.dial. masoa 'loaf, hang around, be idle' Sw. masa (sig) id.; 2.9. Fi. öykätä 'boast, brag, exaggerate' Old Sw. öka 'increase, make larger'.
\end{abstract}

Im folgenden führe ich zu einigen einzelnen finnischen Wörtern deren mögliche germanische Herkunft an. Ein Teil dieser Wörter könnte meines Erachtens zu den sog. älteren germanischen Lehnwörter gehören, also zu Wörtern, die auf eine spätestens urnordische Wortform zurückzuführen sind, während es sich bei einem anderen Teil um jüngere, aus dem Alt- oder Neuschwedischen stammende Entlehnungen handeln kann. Einige dieser Wörter, dies betrifft besonders die Wörter der ersten Gruppe, wurden in der Etymologieforschung bereits früher behandelt. Der Herkunft der Wörter dagegen, die ich als jüngere Entlehnungen ansehe, wurde bislang größtenteils überhaupt keine oder keine nennenswerte Beachtung zuteil.

Etymologien zu den älteren germanischen Lehnwörtern wurden schon seit der Zeit Thomsens bis zum Anfang dieses Jahrhunderts und besonders wieder in den letzten Jahrzehnten in beträchtlicher Anzahl 
vorgelegt; hier wie auch in den Interpretationen der als genuin erklärten Wörter ist sicherlich noch immer einiges zu überprüfen. Offensichtlich ist jedoch, daß ein germanischer Einfluß auf den finnischen Wortschatz schon früh ziemlich stark war, weshalb dieser Ausgangspunkt auch nie vergessen werden darf, sucht man nach der Herkunft der finnischen Wörter. Auf dieser Auffassung beruhen die im folgenden unter Punkt 1 vorgeschlagenen Etymologien.

Die Klärung der jüngeren germanischen Lehnwörter war in den vergangenen Jahren nicht allzu lebhaft. Die Quelle, die das Alt- und Neuschwedische bietet, ist noch keineswegs voll ausgeschöpft: Neben der Hochsprache bieten besonders die finnischen Dialekte reichlich Material, das seiner Erforschung harrt. Eine den Dialektologen wohlbekannte Tatsache ist, daß (alt-)schwedische Lehnwörter in allen finnischen Dialekten auftreten können, manchmal auf engstem Bestimmungsraum, und dabei nicht nur ausschließlich in den westlichen und südlichen Küstengebieten, sondern z. B. auch in den Dialekten von Häme oder in den ostfinnischen Dialekten. In einigen Fällen sind die Dialektwörter ihrer Herkunft nach so eindeutig, daß sie keiner weiteren Deutung mehr bedürfen. Als Beispiel hierfür sollen nur einige Fälle dienen, die meines Wissens bislang nicht in der etymologischen Literatur erwähnt wurden. In einigen Kirchspieldialekten Südwestfinnlands und der Provinz Satakunta kennt man das Wort asko 'Kalklösung oder Aschenlaugelösung' (Vuorela 1979, SMS), vgl. schwed. aska, dial. asko 'Asche'. Stellenweise ist in den Dialekten des Südwestens und andererseits in den nördlichen Dialekten von Häme für äes 'Egge' das Wort harvi (Vuorela 1979, SMS) gebräuchlich, vgl. schwed. harv id. In einigen Kirchspielen der Dialekte des Südwestens und in einem großen Teil der Dialekte von Mittelostbottnien sowie teilweise auch von Nordostbottnien tritt das Wort kartti auf (stellenweise auch karttinen, kaarti) 'unreife Frucht od. Beere' (SMSA), vgl. schwed. kart id. (Die etymologischen Wörterbücher der finnischen Sprache führen zwar derartige Dialekt- oder alte schriftsprachliche Wörter an, s. z.B. SSA s.v. alvi 'Unterboden', arpee$t i$ 'Arbeit', eltari 'Heizer' usw., das Auswahlprinzip bleibt jedoch unklar.) Nicht immer so einfach sind die Fälle, bei denen eine lautliche und/oder semantische Entsprechung zu einem gewissen Grad abweichen kann oder bei denen ein Lehnwort mit einem finnischen Suffix 
versehen wurde oder sich sonst an einen finnischen Wortstamm angepaßt hat. Beispiele für diese Fälle beinhalten besonders die in Punkt 2 angeführten Etymologien.

Als Quellenmaterial dienen vor allem SKES (Suomen kielen etymologinen sanakirja I-IV [Finnisches etymologisches Wörterbuch]) und SSA (Suomen sanojen alkuperä 1 [Die Herkunft der finnischen Wörter]) sowie auch SMS (Suomen murteiden sanakirja 1-3 [Finnisches Dialektwörterbuch]) und SMSA (Suomen murteiden sana-arkisto [Finnisches Dialektarchiv]). Zusätzlich wurden Wörterbücher der ostseefinnischen Sprachen herangezogen, und für die entfernt verwandten finnisch-ugrischen Sprachen besonders UEW (Uralisches etymologisches Wörterbuch). Wichtigste Quellen für die germanischen Sprachen waren die verschiedensprachigen etymologischen und anderen Wörterbücher.

1. Vorschläge zu älteren germanischen Lehnwörtern

1.1. karhe, karho 'Reihe zusammengerechtes Heu', karhita 'Heu in Reihen rechen'

Der Terminus aus dem Bereich der Heuernte karhe 'lange Reihe zusammengerechtes Heu' ist außer in der Hochsprache auch weit in den finnischen Dialekten verbreitet, in denen auch die Formen karhi und karho auftreten. Die entsprechenden Verben sind karhita (karhitse-) 'Heu in karhi rechen' und karhota, karhoa id. Das genau dasselbe bedeutende Substantiv karhe ist auch aus dem Ingrischen und Karelischen bekannt; im Ingrischen lauten die Verben karhottaa und karhutella id., im Karelischen karhita 'Heu rechen'. Daß sowohl karhe als auch karhita schon seit langem zur karelischen Sprache gehören, wird dadurch belegt, daß beide auch im Tverkarelischen auftreten. Im Wotischen ist nur das Verb karotella 'Heu in Reihen rechen' bekannt, in einigen estnischen Dialekten dagegen nur das Substantiv karu '(länglicher) Heuhaufen' (SKES, SSA, SMSA, IMS, KKS; für Estnisch s. auch VMS, wonach karu 'Heuhaufen' in den Dialekten von Ridala, Pärnu und Häädemeeste bekannt ist; vgl. EKMS 1 414: karu 'länglicher Heuhaufen', Häädemeeste und Kihnu). Diese *karhV-Wortfamilie scheint also als Terminus der Heuernte bis auf das Urfinnische zurückzugehen. 
Die etymologischen Wörterbücher der finnischen Sprache erklären den Ursprung des Wortes karhe einigermaßen unterschiedlich. Laut SKES soll karhe aller Wahrscheinlichkeit nach im Zusammenhang mit dem Wort karhi '(Zweig)egge' stehen. Dieses karhi tritt unter den ostseefinnischen Sprachen nur im Finnischen auf (SKES, EEW, SSA), in den Dialekten vor allem in den östlichen und nördlichen Landesteilen. Damit in Verbindung gebracht werden, entweder mit (SKES) oder ohne Bedenken (UEW 646), die mordwinischen und tscheremissischen Wörter für 'Reis, Zweig' (z. B. mordwM. karča und tscher. karša). SSA bezweifelt letztgenannte Verbindung und sieht es als wahrscheinlicher an, daß sowohl karhe als auch karhi mit den Wörtern karhea 'rauh, grob' und karha 'zottiges Haar' in Zusammenhang stehen. Ebenso führt auch Heikki Leskinen (1992: 60) an, daß karhi 'Egge' eine "relativ junge Erscheinung der Savo-Dialekte" sei und sich mit der Besiedelung in das nördlichere Finnland ausgebreitet habe. Diese Auffassung hat auch der Ethnograph Veikko Anttila vertreten (1968: 9-13, 22f.).

Ein Grund dafür, daß SKES (Y. H. Toivonen) die Wörter karhe und karhi miteinander verbindet, mag in den Angaben der alten Wörterbücher liegen. Seit Juslenius (1745) wurde nämlich angeführt, daß karhita auch '(im allgemeinen) harken, rechen, durchkämmen' und karhi(n) '(auch:) Rechen, Harke' bedeuten (die Angaben im SKES für karhi 'Schwendenegge; Egge; Rechen, Harke' entstammen offensichtlich direkt dem Wörterbuch von Lönnrot).

Die Verbindung von karhe mit dem Wort karhea 'rauh, grob' erscheint nicht besonders glaubwürdig, auch wenn ihr möglicherweise die Vorstellung von gröberem, trockenem Heu als Gegensatz zu dem weicheren, eben erst gemähten Heu zugrundeliegen mag. Diese Erklärung würde vielleicht besser zu dem heutigen starken Kleegras passen, als zu dem früher auf Naturwiesen geernteten Heu. Eher ließe sich jedoch aufgrund des Aussehens eine Verbindung von karhe mit dem Wort karha 'zottig, struppig' denken, vgl. karhapää 'Strubbelkopf' (auch als Nachname, s. Mikkonen-Paikkala 1992: 175) sowie olla karhallaan 'struppig, zerzaust sein'. Zudem tritt in Ostbottnien und Nordfinnland stellenweise auch das dazu lautlich nahestehende Wort korho auf, semantisch sowohl 'gewendetes, trockenes Heu' als auch 'Strubbelkopf' (SSA). In beiden Fällen kann es sich jedoch nur um eine lautliche Va- 
riation $a \sim o$ handeln, worauf die lokale Vertretung der Dialekte zu verweisen scheint; ein Einfluß von karha, korho 'struppig' kann in der Wortfamilie karhe dennoch angenommen werden. Die Verbindung der Herkunft dieser beiden Wortfamilien wird wesentlich geschwächt dadurch, daß karha 'struppig' außer im Finnischen nur im Karelischen und auch dort z.B. nicht im Tverkarelischen vertreten ist. Am wahrscheinlichsten ist also, daß der früher sehr wichtige Terminus der Heuernte karhe, karho ebenso wie das Verb karhita hinsichtlich ihrer Herkunft nichts mit den oben angeführten anderen Wörtern auf karhV- zu tun haben.

Zieht man in Betracht, daß der Großteil der alten Termini aus dem Bereich der Heuernte germanischen Ursprungs ist, ist es keineswegs abwegig, daß auch karhe zu derselben Gruppe von Lehnwörtern gehören könnte wie haasia 'Heureiter', hamila 'Heutrage', kärväs 'Trockengestell, Heureiter', kuhja 'Heuschober', kuupano 'kleiner runder Heuhaufen', laes 'Schwaden, Jahn', luoko id., niittää 'mähen', ruko 'Heuhaufen', saatto id., saura id. und takka 'Heubündel' (SKES, Vilkuna 1970: 275, Hofstra 1985: 307f., SSA). Karhe, eine 'Reihe zusammengerechtes Heu', bestand aus zusammengerechtem laes bzw. luoko 'Schwaden, Jahn', und aus einer oder mehreren karhe wurden ruko bzw. saatto 'Heuhaufen' gemacht. Auf germanischer Seite scheint sich auch die mögliche Quelle der urfinnischen Wortfamilie *karhV- aufspüren zu lassen, nämlich das urgerm. Substantiv *karziz und das Verb *karzijan, vgl. ae. cierr m. 'Kehre, Wende' sowie cierran 'kehren, wenden, ändern' (Pokorny 1959: 393: 'wenden, in eine bestimmte Lage bringen', Holthausen) und be-cierran 'turn round, wind, twist' (Borden). Als in gleichem Zusammenhang stehend angesehen werden ahd. kerren 'kehren, wenden' (Pokorny s. v., zuletzt auch Blöndal Magnússon s. v. kerra) sowie isl. kerra 'beugen, rückwärts gebogen sein' und norw. kjerra 'den Kopf aufwerfen bei starkem Wind'. (Zur isländischen Form s. Blöndal Magnússon, der, ebenso wie Torp, damit das norwegische Verb verbindet. Zur Rekonstruktion der germanischen Form s. Pokorny s. v., Blöndal Magnússon s.v., vgl. Holthausen s.v. cierran und EWD s.v. kehren. Dt. kehren 'wenden' und ndl. keren id. sind offensichtlich unterschiedlicher Herkunft, s. Kluge-Seebold, EDW, de Vries 1971.)

Die hier vorgelegte Etymologie geht davon aus, daß die ursprüngliche Bedeutung von ostseefi. *karhV- mit 'wenden; drehen, kehren; 
umwenden, umkehren' verbunden gewesen sein könnte. Wesentlich im Zusammenhang mit karhe und karhita ist wohl gerade das Wenden des Heues, damit es trocknet, und zwar nicht nur während des Zusammenrechens in Reihen, sondern auch später, wie folgende Beschreibung der Heuernte ehemals zeigt: "Mit der Heumahd wurde gewöhnlich früh am Morgen begonnen, wenn das Heu vom Tau aufgeweicht war. Nachmittags wurde es in lange Reihen (pitkiin karheisiin) zusammengerecht, die im Laufe des Tages gewendet wurden. Für die Nacht wurde das Heu zu kleinen Haufen (pieniin rukoihin) aufgeschichtet, die am folgenden Tag wieder zum Trocknen ausgebreitet wurden." (Talve 1979: 64.) Karhita bedeutete also ursprünglich vermutlich 'Heu wenden', und karhe, karho war das Resultat dieses Heuwendens. Im Urfinnischen spezialisierte sich das germanische 'wenden, kehren (allg.)' ausdrücklich zu einem Terminus der Heuernte. Das eigentliche urfinnische Verb für '(um)wenden, (um)kehren' war das in allen heutigen ostseefinnischen Sprachen auftretende (fi.) kääntää. Auf germanischer Seite ist in dieser Wortfamilie auch eine noch deutlichere Bedeutungsverengung zu beobachten: Dem ae. Substantiv cierr entspricht in vieler Hinsicht das neuenglische turn, jedoch bedeutet das in seiner neuenglischen Form char(e) nur 'work by the day at housecleaning' (ODEE, OD), während dagegen im Neunorwegischen der Gebrauch des Verbs nur auf ein bestimmtes Drehen des Kopfes begrenzt ist.

Als das $z$ der germanischen Form *karz-im Urfinnischen durch $h$ substituiert wurde (vgl. z. B. fi. hahtuva 'Flocke' urn. *hazda-, fi. keihäs 'Speer' urgerm. *gaizaz, SSA), entstand *karh-, aus dem schon früh Ableitungen gebildet wurden. Das Verb karhita, karhitseläßt schon aufgrund seiner Form an einer Entlehnung zweifeln: Unter den alten germanischen Lehnwörtern im Ostseefinnischen gibt es recht viele Verben auf -itse, deren Original im allgemeinen ein germanisches Verb auf *jan- ist (Hofstra 1985: 230, s. auch Hakulinen 1979: 285 und Lehtinen 1979: 314). Karhe (<*karhek; auf die Endung *ek weisen z. B. die karelischen Formen, vgl. kar.Tver Nom.Pl. karhiet) könnte ein Derivativum von diesem Verb sein; das führt auch Lauri Hakulinen an (1979: 189), nach dem karhe eine Verbalableitung ist, die "das Ergebnis einer Arbeit oder das Ziel eines Tuns" ausdrückt. Das urfinnische Nomen *karho könnte auch eine Ableitung vom o.g. Verb darstellen, so- 
fern es nicht bereits in der Entlehnungsphase direkt aus der germanischen Stufe gebildet wurde. Es ist auf jeden Fall alt, treten doch in mehreren Sprachen darauf hinweisende Formen auf; karotella im Wotischen und karu (<*karho) im Estnischen sind die einzigen Formen, die in diese Wortfamilie gehören.

\section{2. kuono 'Schnauze, Maul'}

Bereits im Wörterbuch von Juslenius (1745) wird das Wort kuono erwähnt: 'rostrum, os animalis prominens; nos, mula'. Dieses im Finnischen allgemein bekannte Wort hat gleichbedeutende Entsprechungen im Wotischen und Estnischen: wot. koono 'Schnauze, Maul', estn. koon id. (SKES, EEW, SSA). Bei der Erklärung der Herkunft von ostseefinnisch *kōno wurde früher eine Zusammenstellung mit dem mordwinischen Wort für 'Stirn' als unsicher angegeben, mordwE. końa, mordwM. koñä (so z.B. noch SKES). Das Wort fehlt jedoch gänzlich im UEW, und SSA hält eine solche Zuordnung für "unbegründet" (s. auch EEW und Raun 1982).

Eine andere Etymologie für *kōno wurde im Jahr 1981 von Jorma Koivulehto in einem Vortrag vorgeschlagen; danach soll das Wort eine Entlehnung aus der germanischen Form $\left({ }^{*}\right.$ skunō $>$ ) *skonō sein, die im norw.dial. skon f. 'Schnauze, Maul (bes. eines Pferdes)' und fär. skon f. id.; Gesicht' vertreten ist (zur norwegischen Form s. Torp, nach dem die Herkunft des Wortes unklar ist, vgl. jedoch Blöndal Magnússon, s.v. skon $(n) r$; zur möglichen germanischen Etymologie o.g. Wortes kuono s. auch Hofstra 1985: 32, 297). Die Schwierigkeiten, die mit dieser Etymologie einhergehen, betreffen das $o$ der ersten Silbe des germanischen Wortes, dessen normale Entsprechung auch im Ostseefinnischen ein kurzes $o$ wäre. SSA führt diese Etymologie nicht an.

Falls *kōno ein germanisches Lehnwort ist, könnte es sich auch um eine völlig andere germanische Ausgangsform handeln, die in der ersten Silbe ebenfalls ein langes $O$ aufweist: urn. oder urgerm. *gōnō. Auf diese Form geht neuisländisch góna f. 'Schnauze, Maul' zurück. Es hängt ursprünglich mit den isländischen Wörtern gón n. 'Glotzen' und góna 'mit offenem Mund anstarren' zusammen (Blöndal, Jansson, 
Blöndal Magnússon). Damit verwandt sind die auch in größerem Aus$\mathrm{maß}$ in den skandinavischen Sprachen auftretenden Wörter an. gan $\mathrm{n}$. 'Gähnen', gana '(mit offenem Munde) starren, glotzen' (Baetke, de Vries), norw. gan f. 'Rachen, Schlund, Maul' (Torp) sowie schwed. gan 'Kieme; Gaumen; Rachen, Schlund', gana 'gaffen, starren, glotzen' (Hellquist). Zu dieser skandinavischen Wortfamilie scheinen also außer den Wörtern 'Maul, Rachen, Schlund' auch 'starren, glotzen' zu gehören. Vergleichbar mit dem isländischen Substantiv und Verb góna sind z. B. die estnischen Wörter molu 'Maul, Mund' und molutama 'mit offenem Mund gaffen' (EEW).

\section{3. rakentaa 'bauen, aufführen, errichten'}

Das finnische Verb rakentaa hat neben seiner o.g. hochsprachlichen Hauptbedeutung außer der allgemeineren 'machen, fertigmachen' auch solche spezialisierten Bedeutungen wie 'bebauen, beackern', 'reparieren, ausbessern, flicken (Schuhe, Boote usw.)', 'simmen (Netz)', 'das Gewebe anzetteln' sowie '(das Pferd) an-, vorspannen' (SKES), vgl. auch z. B. Juslenius: 'struo, colo, reparo; bygger, brukar, lagar'. Bei zumindest einem Teil dieser Bedeutungen handelt es sich um sichtliche Lehnübersetzungen, vgl. aschwed. byggia 'bebo; bebygga; odla, bruka; befatta sig med' (Söderwall) und ält.neuschwed. bygga u. a. 'laga (nät, vävning osv.)' (Östergren 1919), ebenso im Finnlandschwedischen u. a. 'laga (skodon), lappa (kläder), reparera (fiskbragden)' (Ahlbäck).

Auch im Karelischen verfügt das Verb rakentaa über verschiedene Bedeutungen: 'machen, fertigmachen (bes. Netz, aber auch Haus)', 'das Zimmer mit Türen, Fenstern und Fußboden versehen', 'Fenster und Türen umrahmen' usw. Im Estnischen und Wotischen bedeutet das Verb in erster Linie 'das Pferd anspannen, vorspannen', rakettā im Wotischen auch 'das Haus mit Brettern beschlagen, füttern', rakendama im Estnischen auch '(fig.) anstellen, einsetzen, heranziehen, anwenden; (Wiedemann auch:) einfassen, flicken; sich abarbeiten' (SKES, EEW). Die Hauptbedeutungen der ostseefinnischen Wortfamilie können mit 'aufbauen, errichten' und 'das Pferd an-, vorspannen' wiedergegeben werden. Hinsichtlich letztgenannter Bedeutung ist es interessant festzustel- 
len, daß sie im Finnischen in den allernördlichsten Dialekten auftritt, wo zumindest keine direkte Verbindung zu der Bedeutung der entsprechenden Verben des Wotischen und Estnischen an der Südseite des Finnischen Meerbusens bestehen kann.

Das Verb rakentaa wird für ein Derivativum gehalten (Hakulinen 1979: 265), wobei es sich bei -nta- um ein Ableitungssuffix und dem vorangehenden $e$ um den Stammvokal handelt, wie in den analogen Kausativableitungen hämmentää 'umrühren' (die gleiche Ableitung tritt in den anderen ostseefi. Sprachen auf, außer im Lüdischen, Estnischen und Livischen), kohentaa 'in Ordnung bringen, verbessern' (in den anderen ostseefi. Sprachen, außer im Wotischen und Livischen), korventaa 'abbrennen' (neben dem Finnischen auch im Karelischen, Lüdischen und Wepsischen) oder im Verb oksentaa 'sich übergeben' (entsprechende Ableitungen im Karelischen, Lüdischen, Wepsischen und Estnischen). Man kann also annehmen, daß es im Urfinnischen die Ableitung * rakkenta- gegeben hat, so daß als Wurzel *rakkV-bleibt.

Als mögliches Wurzelnomen wurde manchmal ein Substantiv angeführt (EEW, vgl. SKES), das im Estnischen auftritt: rake (Pl. rakked) 'Pferdegeschirr; Einfassung (des Brunnens)'. Das Wort selbst scheint jedoch offenbar ein - $e$-Derivativum zu sein, so daß es eher aus derselben Wurzel *rakkV gebildet worden sein kann wie *rakkkenta-; vgl. z. B. estn. rike (Pl. rikked) 'Fehler, Schaden' sowie das Verb rikkuma 'verderben, beschädigen' (EEW). Auch im Finnischen und Karelischen tritt das Substantiv rakkine auf, dessen karelische Formen ebenfalls mit den Bedeutungen des Verbs rakentaa im Karelischen zusammenzuhängen scheinen: 'Verfertigung des Netzes', 'Rahmen' usw. Im Finnischen ist rakkine sowohl in der Hochsprache als auch in den Dialekten, in denen es auftritt, in erster Linie ein 'schlechtes, fehlerhaftes Ding (Gerät, Apparat, Werkzeug)'. Auch rakkine ist sichtlich ein Derivativum, sein Zusammenhang mit dem Verb rakentáa gilt jedoch durchaus nicht als gesichert (Hakulinen 1979: 171, SKES s.v. rakkine).

Das Verb wurde aus dem Finnischen und Karelischen in das Lappische entlehnt, wo es über dieselben Bedeutungen verfügt: Lulelappisch 'bauen, aufführen', norwegischlappisch 'machen, fertigmachen, anrichten, formen, aufführen, bauen' sowie inarilappisch 'fertigmachen, bauen'. Die Hauptbedeutungen im Lappischen lauten also 'bauen, fer- 
tigmachen' (SKES). In der etymologischen Forschung wird mit dem Verb rakentaa auch das ungarische rak- (dial. rok-) 'legen, setzen, stellen; (dial.) errichten, bauen' in Zusammenhang gebracht. Auch hier wird davon ausgegangen, daß in dem ostseefinnischen Wort - nta- ein Ableitungssuffix ist. Im SKES ist der Zusammenhang mit dem ungarischen Wort mit einem Fragezeichen versehen, als unsicher wird es auch im UEW "wegen der entfernten Verwandtschaft" (419) angesehen, ebenso z. B. Häkkinen (1990: 255, s. auch Häkkinen 1983: 349; das Wort ist auch nicht im Wörterverzeichnis von Sammallahti enthalten, 1988: 541-551). Noch skeptischer wird die Möglichkeit beurteilt, daß das (ostseefi.-ung.) Wort indogerm. Ursprungs sei, z. B. wird dies weder im SKES noch UEW überhaupt erwähnt; in dieser Deutung wird angeführt “idg. *rag- 'in Ordnung bringen”, "ieur. *raq- 'anordnen”, und damit im Zusammenhang wird erwähnt altind. racayati 'ordnet, verfertigt, bildet, macht zurecht' (vgl. jedoch Pokorny 1959: 863: * rek 'anordnen'; zur Literatur bezüglich der idg. Herkunft des Verbs rakentaa s. Joki 1973: 105f., 197f. sowie MSzFE 524).

Die oben angeführten Fakten scheinen also darauf hinzuweisen, daß das Verb rakentaa erst in urfinnischer Zeit in die Sprache aufgenommen wurde. Auch in diesem Fall kann eine germanische Herkunft für möglich erachtet werden; als Ausgangsform wäre meiner Meinung nach urgerm. *rakjan- anzusehen, das vertreten ist $\mathrm{z}$. B. in dt. recken 'strecken, dehnen' und sich recken 'sich hoch aufrichten, sich strecken, die Glieder dehnen' (Wahrig), got. uf-rakjan 'aufrecken' (Streitberg, Lehmann), ae. reccan 'strecken, ausstrecken' (Holthausen), an. rekja 'winden, (auf-, ab-)wickeln, entfalten, ausbreiten' (Baetke) und aschwed. ræekkia 'räcka, sträcka (sig), nå, räcka till' (Söderwall). Das germanische Verb gehört etymologisch in Zusammenhang mit dem Wort recht; die indogerm. Urform lautet *reg- 'gerade richten, recken, strecken, aufrichten' (Pokorny 1959: 854).

Das von Elmer Seebold aktualisierte Etymologische Wörterbuch der deutschen Sprache von Friedrich Kluge belegt s. v. recken: “die Bedeutung 'recken' ist wohl als 'gerade machen, aufrecht machen' zu verstehen". Genau das ist auch das ostseefinnische rakentaa 'aufrichten, errichten, bauen', aus dem wiederum sich auch die allgemeinere Bedeutung 'zurechtmachen, fertigmachen' entwickeln konnte. Vergleich- 
bar mit rakentaa sind die finnischen Wörter pystyttää 'aufrichten, errichten, bauen' und pysty 'aufrecht', die aus der gleichen Wortfamilie stammen wie pistää 'stechen; stecken; stoßen; hinausstrecken; sich ausdehenen; Zaun machen usw.', dial. pisty 'aufrecht', vgl. auch z. B. weps. pištta, püštta 'stoßen; aufrichten, errichten', pišt 'Zaun', pǚšt 'Wehr' (SKES s.v. pistää und pysty, SVJ). Die Bedeutung des Bauens begegnet außer im Verb pystyttää auch z. B. in den finnischen Dialektwörtern pistos 'ein aus Stangen gefertigtes Zelt', piste 'id.; Zaun, Zaunlatte' (SKES) sowie pistekota (kota 'Hütte, Zelt') 'primitiver, auf vorgeschichtliche Zeit zurückgehender Behausungstyp, dessen Gerippe kegelförmig aus Stangen errichtet ist' (Vuorela 1979).

Erwähnenswert in diesem Zusammenhang ist die Auffassung, die J. Trier in den dreißiger Jahren von den zu o.g. germanischer Wortfamilie gehörenden Wörtern sowie von der ursprünglichen Bedeutung der Wortfamilie vertrat. Obwohl seine Meinung in der etymologischen Literatur später meist ignoriert wurde, führt Jan de Vries sie sowohl in seinem altnordischen als auch im niederländischen etymologischen Wörterbuch an (1962 s.v. rekja und 1971 s.v. rekken). Danach sei der Ausgangspunkt die konkrete Bedeutung 'Flechtwerk, Zaun, Zaunstange' gewesen, die noch erscheint z. B. in mnd. recke, rik, mnl. recke, rec, ric sowie neuniederländisch $r e k$, die alle 'Gestell, Stange' bedeuten (im gleichen Zusammenhang steht auch dt. Reck 'Turngerät'), und die gestützt wird durch solche Wörter wie ae. reced, as. racud und ahd. rachat, deren Bedeutung 'Gebäude, Haus' lautet (über die Herkunft erstgenannter Wörter sind verschiedene Meinungen geäußert worden, s. de Vries 1971, laut EWD ist die "Herkunft ungewiß", vgl. jedoch Kluge-Seebold; die Etymologie letztgenannter Wörter erscheint noch unsicherer, vgl. Pokorny 1959: 64, Holthausen s. v. reced, Lehmann s.v. rohsns).

Das ostseefinnische Verb rakentaa hat andererseits jedoch auch die Bedeutung '(das Pferd) an-, vorspannen'. Obwohl sich diese Bedeutung auch aus der allgemeineren Bedeutung 'fertigmachen' entwickelt haben könnte, könnte hier auch die zentrale Komponente deś germanischen Verbs 'strecken, spannen' zum Vorschein kommen. Aber auch für den Aufbau, z. B. gerade eines Zeltes, ist einerseits das Errichten von Stangen, andererseits das Ausbreiten und Spannen der Felldecken o. ä. um 
die Stützpflöcke herum wesentlich (vgl. dazu z. B. an. rekja 'winden, auf-, abwickeln, entfalten, ausbreiten').

Diese Etymologie einer germanischen Abstammung setzt voraus, daß an das kausative germanische Verb *rak(jan)- im Urfinnischen ein kausatives Ableitungssuffix -(e)nta- gehängt wurde; auch andere urfinnische Ableitungssuffixe treten in alten germanischen Lehnwörtern auf, z. B. maita : maitse- 'schmecken' urgerm. *smakjan-, laitta- 'setzen, legen' urgerm. *lagjan-, kavahta- 'erschrecken; sich hüten' urgerm. * $\chi a w e(j a) n$ - usw. (s. Hofstra 1985: 237-241). Das germanische *-kwird im Urfinnischen erwartungsgemäß durch *-kk- vertreten.

1.4. tana- in "olla tanassa" 'ausgestreckt sein', tanakka, tanea 'stämmig, untersetzt, steif, fest, stark'; taana 'still, träge'; tauno id.

Das Wörterbuch der finnischen Gegenwartssprache (Nykysuomen sanakirja [NS]) führt in der Regel neben dem Wort tanakka auch das seltenere Adjektiv tanea 'fest, stark, stämmig, gedrungen' an. In den Dialekten ist erstgenanntes weit verbreitet, u. a. wird im Dialekt von Parikkala tanakka so erklärt, daß es sich dabei um jemanden (oder etwas) handelt, der sich weder leicht beugt noch biegt: Sowohl ein Mensch als auch eine Angelrute kann tanakka sein. Das Adjektiv tanea 'stämmig, fest, steif' wiederum tritt in erster Linie in den Ostdialekten sowie in den südöstlichen Dialekten von Häme, seltener in Süd- und Mittelostbottnien auf, z. B. "Tanneet ne on nuo sarkaset housut, seisovat ilema miestäki" ('steif sind sie, diese Lodenhosen, stehen auch ohne Mann', Mikkeli); das Wort hat in den Dialekten stellenweise auch die Bedeutung 'gelassen, gesetzt'. Hauptsächlich aus den nördlichen finnischen Dialekten wurde noch das Adjektiv tanu 'stämmig, untersetzt; fest; zuverlässig' 'aufgezeichnet. Außerhalb des Finnischen ist das Adjektiv tanakka, tanakko, tanakku 'fest, stark; steif' nur im Karelischen bekannt. (SKES, SMSA.)

SKES führt diese Wörter s.v. *tana: tanaan, tanassa 'ausgestreckt, gerade' an, welche Formen stellenweise sowohl in den Dialekten Ostals auch Westfinnlands auftreten. Nach dem NS bedeutet tanassa 'robust, kräftig, steif, starr (nach vorne) gerichtet, ausgestreckt', z. B. 
"Sotilaat syöksyivät rynnäkköön pistimet tanassa" ('Die Soldaten stürmten zum Angriff mit gefälltem Bajonett'), "Siilin piikit ovat tanassa kaikille tahoille" ("Die Stacheln des Igels sind in alle Richtungen aufgerichtet'); als Terminus technicus aus dem Turnsport werden Komposita mit tana- als erstem Element angeführt: tana-asento 'Haltung, bei der ein Bein so hoch wie möglich gerade nach vorn gestreckt wird' und tanakäynti 'Schritt, bei dem ein Bein, ohne es zu beugen, nach vorn gehoben oder geschleudert wird'.

In den alten finnischen Wörterbüchern erscheinen die Adjektive erstmalig bei Eurén (1860): tanakka 'stark, fast, styf, pålitlig, ihärdig', tania 'stadig, stadgad, bastant, styf'. Das Wort tana findet sich bereits bei Renvall (1826): "positura rei ad quid proclivis; bereite Stellung etwas zu thun, e.c. jalka 1. käsi on tanassa pes est positus 1. paratus ad currendum, manus ad caedendum, pani kätensä tanaan". In Verbindung mit dem Wort tana steht wahrscheinlich auch tanottaa 'extendo longum quid, hinc mentem intendo, desidero; ausstrecken, sehnen, begehren', ebenfalls bei Renvall, z. B. "tanotan taiwaaseen" ('ich sehne mich nach dem Himmel'). Renvall führt dies in demselben Zusammenhang an wie tanottaa 'mit langer Birkenrinde umwickeln', vgl. tano 'langer Streifen von Birkenrinde'. In gleicher Weise behandelt auch z. B. Lönnrot diese Verben zusammen: "tanotta v.fact. (tano) omvira med näfverremsa, omlinda; uttänja, utsträcka; längta till”. Renvall entnahm dieses Verb dem als Manuskript überlieferten Wörterbuch von Ganander (1786-87), wo auch das Beispiel dasselbe ist ("Tanoitta Taiwahasen"; das Wort fehlt in der Untersuchung von Hormia über die Quellen Gananders, 1951). Ganander behandelt jedoch das Verb tano(i)ttaa getrennt von dem Wort tano 'Streifen von Birkenrinde' und erklärt das Verb so: "Tanoitan, -ttaa - V.N. sträcker ut transl. längtar”.

Sowohl lautlich als auch semantisch wäre für die oben behandelte Wortfamilie tana als ursprüngliche Form geeignet urn. *bana, dessen Nachfolger norw. tan n. 'Ausspannung, Ausdehnung, Ausstrecken' (Torp) und isl. pan n. 'Streckung, Ausdehnung' (Blöndal Magnússon) sind; in dieselbe Wortfamilie gehören noch u.a. agutn. pan $\mathrm{f}$. 'Sehne', gotl. tan id. (Gustavson, Hellquist s.v. tanig) (semantisch vgl. z. B. fi. jänne 'Sehne' und jäntevä 'stark, kraftvoll', jänt(t)erä 'stark, stämmig', $\mathrm{SSA}$ ), an. pon f. 'Gerüst, Rahmen zum Spannen u. Trocknen (von Fel- 
len)' (Baetke, de Vries), norw. tane 'Spannbrett', tona 'id.; Spannung' (Torp), an. Benja '(aus)dehnen, (aus)spannen' (Baetke), aschwed. parnia 'tänja, spänna' (Söderwall), schwed. tänja '(aus)dehnen, strekken, spannen' (vgl. o. tanottaa, das Lönnrot mit 'uttänja' übersetzt) sowie das schwedische Adjektiv tanig 'hager, mager; sehnig; zäh' (Hellquist).

Im SKES werden im Zusammenhang mit der oben behandelter Wortfamilie tana- auch solche, nur stellenweise auftretenden (hauptsächlich in den finnischen Westdialekten) Verben angeführt, die ein langsam ausgeführtes Tun ausdrücken, wie tania 'langsam sein, zögern, trödeln; langsam und mühsam sich fortbewegen', tanitella, tanailla 'zaudern, zögern'. Im SKES bleibt offen, ob diese Verben ebenfalls im Zusammenhang mit dem Wort tana stehen. Andererseits werden taana 'langsam, träge', taano 'ruhig, sanft' und taanu 'ruhig, gelassen' im SKES völlig gesondert behandelt, die in den südöstlichen und den Dialekten von Savo auftreten und in ihrer Bedeutung nahe an die o.g. Verben kommen, zum Teil auch an die Bedeutung des Adjektivs tanea 'gelassen, gesetzt' (das ebenfalls hauptsächlich in den östlichen Dialekten bekannt ist). Die mögliche Entlehnungsquelle sowohl für die Verben als auch für die Adjektive kann in dem schwedischen dialektalen Verb tana 'schläfrig sein' sowie dem Adjektiv tanig, tani(g)er 'schläfrig, müde' (Rietz) liegen. Nach dem schwedischen etymologischen Wörterbuch von Hellquist könnten diese Wörter ursprünglich mit dem o.g. schwedischen Adjektiv tanig 'hager, mager; sehnig; zäh' verwandt sein, das wiederum mit dem schwedischen Verb tänja in Zusammenhang steht; Blöndal Magnússon verbindet in seinem isländischen etymologischen Wörterbuch s.v. tan n. 'Zwerchfell' (< altdän. $\tan (e)$ id.) das schwedische dialektale Adjektiv taniger 'schläfrig, müde' direkt mit dieser Wortfamilie.

Diese letztgenannten Wörter der östlichen Dialekte wurden offenbar auch beeinflußt durch die in denselben Dialekten auftretenden Adjektive tauna 'gelassen, ruhig' und taunea id. Schon in den alten Wörterbüchern seit Juslenius erscheint tauno 'zahm', was Ganander und Renvall in ihren Wörterbüchern als solches übernehmen, während nach Lönnrot wiederum tauno = tauna 'spak, stilla, sedig, saktlig'; dasselbe bedeutet auch das taunea im Wörterbuch. Schon vorher hatte Eurén in seinem 
Wörterbuch das Wort tauna 'spak, stilla, saktlig' angeführt. Die Bedeutung 'zahm' ist für das Wort in den Dialekten nicht belegt. Dieses Wort wurde früher als germanische Entlehnung angeführt, doch scheinen die Erklärungen nicht sonderlich gelungen: Das angenommene urn. *tabna 'zahm' ist Theorie, die Verbindung des Wortes mit an. tafn n. 'Opfer; Fang; Nahrung' wiederum ist semantisch bedenklich (über die Etymologie s. SKES, de Vries, Collinder 1932: 50; über die Herkunft von tafn zuletzt Lühr 1988: 335 und Blöndal Magnússon). Falls tauna eine germanische Entlehnung ist, könnte meiner Meinung nach jene skandinavische Wortfamilie in Frage kommen, zu der an. pagna 'still werden, verstummen, schweigen', aschwed. paghna id., adän. tavne id., schwed. dial. tagna id. (<*ba(g)anōn), an. pogn 'Schweigen', norw. togn id. (Baetke, de Vries, Torp, Rietz, Blöndal Magnússon) gehören. Das in den germanischen Lehnwörtern dem $n$ vorangehende $g$ wurde durch $u$ ersetzt sowohl in den älteren (fi.dial. uunas 'Esse; Ofen' urgerm. *ugnaz, s. SKES) als auch in den jüngeren Fällen (uuni 'Ofen' aschwed. ughn id., eine jüngere Entlehnung des gleichen Wortes wie o.g; ebenso Maunu 'Magnus', siunata 'segnen', vaunu 'Wagen' usw., s. Rapola 1966: 232).

2. Vorschläge zu jüngeren germanischen Lehnwörtern

\section{1. ahdike, ahturi, atturi 'nachmittags auf dem Feld verzehrter Imbiß'}

In dem volkskundlichen Wörterbuch von Toivo Vuorela (Kansanperinteen sanakirja, 1979: 11) wird das in Häme auftretende Wort ahdike 'auf dem Heu- oder Getreidefeld abends um sechs verzehrter Imbiß' angeführt, wie auch das gleichbedeutende Wort atturi. Die Information geht zurück auf eine Umfrage in der Zeitschrift Sanastaja ("Wortsammler”) aus dem Jahr 1935 (Nr. 23, S. 5), wo angeführt wird, daß beide Wörter in Kalvola gerade in dieser Bedeutung verwendet werden.

Nach dem Wörterbuch der finnischen Dialekte (Suomen murteiden sanakirja) handelte es sich bei ahdike (dial. ahrike, ahlike) 'ein nachmittags gegen fün Uhr auf die Wiese oder das Getreidefeld gebrachter Imbiß, normalerweise Kaffee und belegte Brote' um ein Wort, das un- 
gefähr in der Gegend zwischen Tampere und Hämeenlinna verwendet wurde, während atturi 'Imbiß' wiederum in den davon südlichen Dialekten von Häme bekannt war. Zusätzlich dazu jedoch wird im Wörterbuch auch die Form ahturi 'nachmittags auf dem Feld verzehrter Imbiß' (Sahalahti, Vanaja) angeführt. Das relativ kleine Verbreitungsgebiet der Wörter erklärt offensichtlich den Umstand, daß unter den Wörterbüchern nur in dem von Lönnrot in dem Ergänzungsheft die Form atturi 'mellanmål' angeführt ist.

Der Herkunft dieser Wörter der Dialekte von Häme wurde bislang keine Aufmerksamkeit geschenkt. Vielleicht würden sie einfach mit dem Verb ahtaa 'füllen, stopfen' verbunden werden, das stellenweise auch in den Dialekten von Häme die Bedeutung 'sich voll stopfen, fressen usw.' (SMS) haben kann. Diese Erklärung erscheint jedoch nicht besonders gut zu dem nachmittäglichen leichten Imbiß zu passen, und überdies ließe es die aus vielen Kirchspielen aufgezeichnete Form atturi unklar. In den Wörtern scheinen auf jeden Fall die auf Ableitungssuffixe verweisenden Endungen -ike und -uri aufzutreten, die Wurzel ist aht- und att-.

Zieht man in Betracht, daß dieser Imbiß vorzugsweise nachmittags oder am frühen Abend genossen wurde, könnte als Ursprung dieser Wörter gut das schwedische Wort afton 'Abend, Nachmittag' angenommen werden, das in der älteren Sprache auch die Bedeutung 'Abendbrot, Imbiß am Abend' (IRSSK) aufwies, vgl. aschwed. aptan, aftan, apton, apten, apt- 'eftermiddag, afton' (Söderwall) und schwed.dial. (z. B. gotl.:) afte, aftä, aft-, aftu- (agutn. aftun) id. (Gustavson), finnlandschwed. afton, afto, aftu id. (Ahlbäck, Vendell). Gerade mit Hilfe des Schwedischen ließe sich die in den finnischen Wörtern auftretende Dualität - $h t$-, - tt- erklären, da es sich sich in diesem Fall ebenso verhält wie z. B. in dem schwedischen Lehnwort luhti, lutti 'Boden, Oberlaubenspeicher', vgl. aschwed. loft, lopt, lupt id. (s. SKES s.v. luhti). In Häme, wo nicht kontinuierlich Kontakt zur schwedischsprachigen Bevölkerung bestand wie in den finnischen Küstendialekten, hat sich das z.B. vom Gesinde eines Gutshofs erlernte Wort den von früher her bekannten Derivationstypen angepaßt. Es scheint, als sei die Ausgangsform ein schwedisches Wort gewesen, in dem kein $n$ auftrat, da aufgrund der Form afton eher eine Form mit der Endung -na zu erwarten 
wäre, vgl. z. B. fi. peruna 'Kartoffel', päärynä (< aschwed., ält. neuschwed. pärun, schwed. päron 'Birne'), tatsuuna 'Eisenbahnstation' (< schwed. station id.) usw.

\section{2. aunehtia 'beneiden'}

Das Wort aunehtia 'einen beneiden, auf einen neidisch sein' wurde aus den den Südteil des Päijänne-Sees umgebenden Kirchspielen aufgezeichnet, z. B. "älä sinä nyt aunehli niit min rahojai" ("beneide mich nicht um mein Geld') (Hollola), "ei sinunkua tarttis aina toisesta puhuva ja aunehtia" ("du bräuchtest auch nicht immer über einen anderen reden und ihn beneiden') (Sysmä) (SMS).

Die Ausgangsform des Verbs könnte meiner Meinung nach das schwedische avunda(s) 'beneiden' sein, das z. B. im östlichen Uusimaa in den finnlandschwedischen Dialekten auch in der Form avunna 'mißgönnen, beneiden' (Ahlbäck) auftritt. Von dort wanderte es offenbar weiter nach Norden und wurde gleichzeitig "fennisiert": Aus der Form avunna entstand in den finnischen Dialekten nach dem Schwund von $v$ (vgl. avuttaa > auttaa 'helfen') die Form *aunna, und -nn- nach dem so entstandenen Diphthong wurde gekürzt (zu den Dialekten von SüdostHäme vgl. Lindén 1942: 155-156). Morphologisch gesehen ist aunehtia offenbar unter dem Einfluß des gleichbedeutenden Verbs kadehtia entstanden, vgl. auch das lautlich nahestehende ahnehtia 'gierig, geizig sein'. Analog zu kadehtia 'neidisch sein' - kade 'neidisch', ahnehtia 'gierig sein' - ahne 'gierig' wurde in einigen Kirchspielen (Joutsa, Kuhmoinen) das Adjektiv aune 'neidisch, mißgünstig' (SMS) gebildet.

\section{3. hiiata, hiiautua '(ver)zögern', hiimailla 'zögern, zaudern'}

Das finnische dialektale Verb hiiata verfügt über etwas voneinander abweichende Bedeutungen, von denen sich als wichtigste jedoch 'betrügen' und 'verzögern, zögern' abzeichnen. SSA führt an, daß von diesen Verben hiiata 'betrügen, prellen', das in den südöstlichen Dialekten von Häme bekannt ist, eine Entlehung des schwedischen Dialektverbs hi(a) 
'betrügen, anführen' ist (in diesem Zusammenhang wird auch aschwed. higha id. angeführt; zur Etymologie s. Karsten 1943/44: 123). Dagegen ist es laut SSA unsicher, ob in diesen Zusammenhang auch hiiata 'verzögern, aufhalten, hindern; schleppen; hinken' gehört; gleichfalls wird in dem Wortartikel vermutet, daß dieses Verb zumindest zum Teil von dem schwedischen Verb hejda 'zum Stehen bringen, aufhalten, hemmen, mäßigen' abstammt, zum Teil “vielleicht deskr[iptiven] Ursprungs" ist. Am Ende des Wortartikels wird auch auf das Wort hiivata 'hieven, heben, schleppen; werfen' (< schwed. hiva id.) verwiesen. Zu letztgenanntem hat das Verb hiiata eine offensichtliche Verbindung hinsichtlich seiner Bedeutung 'schleppen', vgl. auch fi. hilata 'schleppen' sowie fi. hinata 'schleppen, ziehen'. Dagegen scheint eine Verbindung des Verbs hiiata zu dem schwedischen Wort hejda lautlich gesehen zweifelhaft.

Laut SMS ist das Verb hiiata in verschiedenen finnischen Dialektgebieten bekannt; es verfügt über die folgenden Bedeutungen, die meines Erachtens mit o.g. Bedeutung '(ver)zögern' verbunden werden können (hier in der im SMS angeführten Reihenfolge): 1. 'hemmen, hindern, verzögern', 2. 'aufschieben, hinausschieben; sich verspäten, sich verzögern', 3. 'langsam gehen, sich schleppen; hinken', 4. 'verlangsamen, Geschwindigkeit vermindern, bremsen', 5. 'schleppen'. Ferner tritt das Verb hiiautua, z. B. auch hiiantua, hiiahua, hiiauta, 'zögern, zaudern, sich verzögern, sich verspäten; langsamer od. träger werden' auf, das aus denselben Dialekten bekannt ist wie die oben unter Punkt 2 angeführten Beispiele: aus den südöstlichen Dialekten, aus Nordostbottnien sowie aus Värmland.

Das Verb hiiauta 'langsamer werden, gehemmt werden (im Wachstum)' taucht wiederholt in Almanachtexten aus der zweiten Hälfte des 18. Jahrhunderts (VKS) auf, deren Verfasser auch andere nordostbottnische Dialektzüge aufweisen. Nach dem Wörterbuch von Renvall bedeutet hiiata 'verzögern'; angeführt werden hier auch das gleichbedeutende Verb hiiailla sowie die Verben hiiantua und hiiauta 'verzögert werden'. Nach Lönnrot bedeutet hiiata u.a. 'hindra, fördröja, uppehålla'.

Das Verb hiiata ist eindeutig eine Entlehnung aus dem Skandinavischen, z. B. weist das schwedische Verb hi(a)- in den Dialekten auch solche Bedeutungen auf wie '(ver)hindern, hemmen, aufhalten' (Rietz, 
Gustavson), 'aufhören (Unwetter)' (Vendell). In dieselbe Wortfamilie gehören u. a. norw. hia 'verzögern, auf die Länge ziehen' (Torp), 'verschieben, verzögern, hinauszögern' (Hustad) sowie isl. hía 'zaudern, zögern; abwarten usw.' (Blöndal Magnússon); die urgerm. Form des Verbs lautete *hījōn (Blöndal Magnússon).

In den finnischen Dialekten tritt vereinzelt auch das Verb hiimailla 'zögern, zaudern' auf; es erscheint in einigen Kirchspielen der Järviseutudialekte in Ostbottnien (Alajärvi, Vimpeli, Lappajärvi). Das ein langsames Gehen bedeutende Verb hiimoa wiederum ist aus zwei der nördlichsten finnischen Dialekte (Kittilä, Raisi) bekannt. Von ihnen stammt zumindest hiimailla auch aus dem Schwedischen, vgl. finnlandschwed. hima 'zaudern, zögern, langsam sein' (Vendell), 'trödeln, langsam und träge sein, auf sich warten lassen' (Ahlbäck), das in den finnlandschwedischen Dialekten Südostbottniens die Bedeutung 'passiv und in Gedanken versinkend sitzen' (Ahlbäck) aufweist. Ein entsprechendes Verb tritt bereits im Altnordischen auf: híma 'müßig, gedankenlos herumstehen od. -schlendern, trödeln' (Baetke, de Vries), vgl. isl. híma id. (Jansson, Blöndal Magnússon). Im Finnischen hat sich das Verb an die genuinen Derivate angepaßt. Mit den Svezismen der Järviseutudialekte hat sich Tuomo Tuomi bereits früher beschäftigt (1990: 194-205); zu ihnen gehört somit auch das Lehnwort hiimailla.

Es stellt sich noch die Frage, in welchem Ausmaß auch einige andere verwandte Bedeutungen der finnischen Verben mit hii- genuin sind oder in wieweit es sich um Entlehnungen handelt, vgl. urgerm. ${ }^{*}$ hijjōn. Beispielsweise wäre es möglich, das finnische hiipua 'langsam zu brennen aufhören', in den Dialekten von Häme auch 'einschlafen, gefühllos werden (Glieder)', als Derivativum mit $p u$ vom Stamm hii- zu erklären, besonders angesichts der in ihrer Bedeutung nahestehenden Verben wie häipyä 'verschwinden', luopua 'auf etwas verzichten, aufgeben, aufhören', uириa 'müde werden', viipyä 'verweilen, zögern', voipua 'ermüden, enkräftet werden', nach deren Muster sich hiipua hätte bilden können. Vielleicht könnte auch sogar fi. hiihtää 'sich auf Skiern fortbewegen; sich schleppen, langsam gehen' (NS; dial. hihtää, hi(i)htaa usw. id.), ursprünglich offenbar 'langsam gehen, schlurfen' (T. Itkonen 1957: 124f., 159f.), ein Derivativ mit $h t A$ von derselben Wurzel sein (vgl. z.B. SKES s.v. viehtää 'locken, verleiten', in dem ein Derivativ 
vom Verb vie- (viedä) 'bringen, leiten' vermutet wird). Nach SSA ist hiihtää "vermutlich späten deskriptiven Ursprungs", was zumindest hinsichtlich von "spät" nicht der Fall sein kann, da das Verb außer im Finnischen in allen östlichen ostseefinnischen Sprachen einschließlich des Wepsischen auftritt.

2.4. hinnerikki, hinterikki 'Hindernis, Ungelegenheit, Störung, Beschwerde', hinnertää 'hindern'

In den Südteilen der Südostdialekte wurde das Wort hinnerikki verwendet, z. B. "huomillall lupasiit meikii reisumiehet tulla kottii, jossei mittää hinnerikkii satu" ('auch unsere Reisenden haben versprochen, daß sie morgen abend nach Hause kommen, sofern kein Hindernis eintritt') (Kivennapa). Als Parallelform trat auch hinterikki auf: "satteethan nä nyty o tehneet hinterikkii heinäteolle" ("die Niederschläge waren jetzt ein Hindernis für die Heuernte') (Kuolemanjärvi) (SMS).

Hinnerikki und hinterikki entstammen sichtlich dem schwedischen Wort hinder 'Hindernis'. An die Endung trat im vorliegenden Fall das finnische - $i k k i$. In einigen Kirchspielen der südöstlichen Dialekte begegnet auch eine Anpassung an eine andere Endung: hinnerrys 'Hindernis, Verspätung usw.'. Desgleichen tritt hinnerrys id. auch in völlig anderen, nämlich den Dialekten von Westerbotten auf, wo es eindeutig mit dem Verb hinnertää 'hindern' (SMS) der gleichen Dialekte verbunden ist. Dieses hinnerrys 'Hindernis' taucht auch einmal in der alten finnischen Schriftsprache auf, in einem Gesetzestext (VKS) aus dem Jahr 1673; in den Wörterbüchern findet sich hinnertää zum ersten Mal bei Lönnrot: 'förlama, hindra; genera, skada', ebenso hinnerrys 'hinder, men, olägenhet, skada'. Lönnrot gibt zu verstehen, daß hinnertää in denselben Zúsammenhang gehöre wie hinterä 'svag, späd, spenslig; slapp, eftergivande', was als Erklärung der Herkunft nicht gelungen wirkt. Direkt dem schwedischen hindra 'hindern, verhindern, hinderlich sein; hemmen; einem unbequem, lästig sein; einen belästigen, beengen, genieren' entstammt hintrata id. in den Dialekten von Turku, Perniö und Vihti; in den Dialekten von Südostbottnien wird dasselbe Verb verwendet für eine langsame, beschwerliche, mühsame Bewe- 
gung, Fortbewegung o. ä. Diesem Dialektwort hintrata wurde bereits früher Aufmerksamkeit zuteil (Karsten 1943/44: 124, Grönholm 1988: 61). In einigen Kirchspielen im Süden des finnischen Häme wird das Verb auch für das Abnehmen der Sehkraft verwendet: "minuj jo silmäni pahastih hintraa" ("meine Sehkraft/Augen hat/haben sich schon sehr verschlechtert') (Hausjärvi) (SMS).

\section{5. illata 'billigen, erlauben'}

Wenigstens seit dem Anfang dieses Jahrhunderts ist das besonders von Kindern und Jugendlichen in Tampere und seiner näheren Umgebung verwendete Verb illata 'billigen, erlauben' bekannt, wobei es in der Regel mit einer Negation verbunden ist: "ei illata" ('nicht erlaubt sein'). Es wurde besonders bei verschiedenen Spielen verwendet, wenn auffiel, daß jemand gegen irgendeine Regel verstieß. Es konnte jedoch auch in anderem Zusammenhang auftreten. Die Schriftstellerin Esti Heiniö aus Tampere erzählt in der Kolumne "Kangasala ja minä" ('Kangasala und ich'), die sie 1956 im Alter für die Zeitschrift Kangasalan Joulu schrieb, wie sie seit ihrer frühen Kindheit, wo sie jeden Sommer in Kangasala verbrachte, als kleines Mädchen gern zu den Leuten von Kangasala gehören wollte, "doch das wurde nicht gebilligt, ich blieb eben eine aus Tampere" ("mutta ei sitä illattu, oli oltava vain tamperelainen").

Im Finnischen Dialektarchiv finden sich zu diesem Verb Angaben außer aus Tampere nur noch aus einigen nahegelegenen Kirchspielen (Kangasala, Lempäälä, Teisko). Danach wird das Wort im allgemeinen als zur Kindersprache gehörend bezeichnet; Beispiele für den Gebrauch sind u. a. "Tota ei illata" ("das ist nicht erlaubt'), "Ei noi pojjaat illaa muuta kun omat lyäntinsä” ('diese Jungen lassen keine anderen als ihre eigenen Schläge gelten [im Ballspiel]') (Teisko).

Hinter dem Verb illata steht vermutlich das schwedische gilla 'genehmigen, billigen', finnlandschwed.dial. gill( $a$ ), jill( $a$ ) id. (Ahlbäck), vgl. (IRSSK:) "något fusk gillas (gills) inte" 'Mogeln ist nicht erlaubt', (beim Spielen: “det gillas (gills) inte” 'das gilt nicht!'. Bei der Entlehnung schwand $j$ am Anfang der schwedischen Form jilla. Dieser 
Schwund entspricht der Erwartung, da die Phonotaktik des Finnischen nur schwer Wörter auf $j i$ - zuläßt; die wenigen Wörter der finnischen Schriftsprache auf $j i$ - sind späte Entlehnungen und auch dann Fachtermini, wie z.B. jiddis, jiujitsu usw. (S. auch Wiik 1969: 224, Karlsson 1983: 131. Das einzige derartige Wort in SKES und SSA ist das in den Dialekten des äußersten Nordens auftretende jiekiö 'an der Mitte eines Stieles befestigtes Schabeisen zur Lederbearbeitung', eine Entlehnung aus dem Lappischen, vgl. z. B. norwegischlappisch jiek'ko id. Das Wort tritt in denselben finnischen Dialekten neben der Variante jiekiä auch in den Formen iekiö, iekko auf.) So hat also das Wort, das in Tampere aus der Sprache der schwedischsprachigen Kinder übernommen wurde, im Finnischen die Form illata erhalten.

\section{6. inttää 'widersprechen, bestreiten'}

Das finnische Verb inttää 'zäh an seiner Meinung festhalten, unaufhörlich wiederholen; widersprechen, bestreiten, leugnen' (NS) ist weder im SKES noch im SSA behandelt worden. Hakulinen (1979: 319) hält das Wort für genuin (s. auch T. Itkonen 1980: 108). Im NS sind auch die Verben inttailla 'sich bedenken, sich widersetzen' und intellä 'zanken, streiten' angeführt. Das Verb inttää 'widersprechen, hartnäckig eine Tat, eine Aussage usw. leugnen' ist auch in den Dialekten gut bekannt, Aufzeichnungen darüber existieren besonders aus Ostfinnland. In den Westdialekten ist intata (inttaa-) häufiger, oft in der Konnexion intata vastaan 'widersprechen, bestreiten, zanken': "se inttaa aina vastaan, puhuu millai hyvänsä” ('er widerspricht einem ständig, man kann zu ihm sagen, was man will') (Kiikoinen). Vereinzelt gibt es aus verschiedenen Gegenden auch Dialektangaben über die Verben inttailla 'sich widersetzen' und intellä 'streiten; widersprechen', z. B. "mee nyt äläkä inttele" ('gehe jetzt und sträube dich nicht so') (Lestijärvi) (SMSA).

Die erste Eintragung in einem Wörterbuch stammt von Ganander: intätä 'nekar, säger emot, giör til intet; pernego', und das gleiche Verb findet sich bei Renvall: intätä 'verneinen, sich widersetzen, vereiteln'. Ganander bringt zusammen mit der Behandlung des Wortes auch die Etymologie: "a Svec. intet". Dies führt auch Renvall an, versieht es 
jedoch mit einem Fragezeichen. Seitdem wurde diese Möglichkeit einer Herkunft nicht wieder aufgenommen, obwohl sie durchaus beachtenswert ist. Die Ausgangsform könnte die schwedische Negation inte sein, vgl. aschwed. inthe, inkte, ingte usw., sowie seine umgangssprachliche Form int, vgl. z. B. "No int, sanoo Marttilaanen" ('also nein, sagt/e Marttilainen') (Vähäkyrö, SMSA). Eindeutige Derivativa des Wortes int(e) könnten zumindest intata, intätä sein, aus dem wiederum inttailla abgeleitet wurde, vgl. z. B. jahka 'wenn, so bald als, wenn (nur)', jahkata 'mit heimlicher Mühe etwas thun', jahkailla 'ein und dasselbe wiederholen' (Renvall 1826; s. auch Hakulinen 1979: 259). Morphologisch gesehen könnten die finnischen Formen also etwa auf ähnliche Weise entstanden sein wie z. B. siettää 'duzen' (sie 'du') oder kohata (kohtaa-) ' "kohta" sagen' (kohta 'gleich, bald'), die von Inkeri Tuovinen (1931: 57) aus den finnischen Dialekten im schwedischen Westerbotten im Jahr 1930 aufgezeichnet wurden. Ähnlich verhält es sich auch mit dem alten Derivativ evätä 'verweigern, ablehnen, -streiten', das aus der Partizipform des Negationsverbs epä 'nicht' abgeleitet wurde; vgl. auch den Neologismus aus dem 19. Jahrhundert eittää 'verneinen, leugnen, bestreiten', der wiederum aus der Negation $e i$ 'nein, nicht' gebildet wurde (SSA). Entsprechende Verben können in den diversen Sprachen gebildet worden sein, erwähnt seien hier nur z. B. an. neka 'verneinen' ( $\leftarrow$ ne Adv. 'nicht'), schwed. neka und förneka 'verneinen, leugnen, bestreiten' oder an. neita “"nein” sagen; ausschlagen, verschmähen, ablehnen; verweigern' ( $\leftarrow$ nei Adv. und Interj. 'nein'; Baetke, de Vries), aschwed. neta 'förneka' (Söderwall).

\section{7. kyönätä 'berücksichtigen, bemerken; einsehen'}

Hauptsächlich in den Zentraldialekten von Häme, aber stellenweise auch in den benachbarten Dialekten begegnet das Verb kyönätä (dial. kyänätä) 'berücksichtigen, beachten, bemerken, wahrnehmen; einsehen, auffassen', besonders in der Negationsform "ei kyänää". In einigen Kirchspielen ist es nur bekannt in der Redensart "kyllä se kuulee, muttei se kyänää”, was entweder durch 'so tun, als ob man etw. nicht hören, bemerken würde' oder durch 'nicht verstehen' interpretiert wer- 
den kann. Ein Kolumnist der in Tampere erscheinenden Zeitschrift Tammerkoski schreibt im Jahre 1965: “- herrasmies katselee virtaavaa vettä siinä ikkunan tuntumassa eikä kyönää vähääkään tyttöjen pyynnölle" ("der Gentleman betrachtet das strömende Wasser dort in der Nähe des Fensters und achtet nicht auf die Bitten der Mädchen').

Dieses kyönätä entstammt offenbar dem schwedischen Verb skönja 'wahrnehmen, bemerken', das im älteren Neuschwedisch auch in der Form sköna 'id., einsehen', sköna på 'auffassen, begreifen' (Dahlgren 1914/16) auftrat und das die Ausgangsform für das Wort kyönätä gewesen sein könnte, vgl. aschwed. skonia 'undersöka, se, ha för ögonen, förstå sig på’ (Söderwall, s. auch Hellquist s. v. skön). Das anlautende $s k$ des Schwedischen wurde auch in vielen anderen Fällen im Finnischen durch $k$ ersetzt (s. Streng 1915: 296 und Grönholm 1988: 202), dem schwedischen langen $\ddot{o}$ kann im Finnischen $\ddot{u} 0$ entsprechen, wie z. B. in den Wörtern kyökki 'Küche' (< schwed. kök id.), kyörätä 'verjagen' ( $<$ schwed. köra id.) usw. (SSA).

\section{8. masoa 'trödeln, faulenzen'}

In Häme und in Satakunta wurde das Verb masoa verwendet, wenn sich jemand unnötig lange verspätete oder wenn jemand bummelte oder faulenzte (SMSA, Virtaranta 1946: 281, 366 sowie 1956: 780): “Tyrväässä sanotaan laiskana olemista masomiseksi”' ('In Tyrvää wird Müßiggang mit masominen bezeichnet') (SMSA).

Dieses Verb ist sicherlich eine Entlehnung des schwedischen Wortes masa (sig) 'trödeln, schlendern', vgl. finnlandschwed. dial. masa id. (Vendell). Zur gleichen Wortfamilie gehören schwed. mas m. 'träger, fauler Mensch' und mas n. 'nachlässige Arbeit' (Dalin) sowie masig 'träge, langsam, untauglich' (Hellquist s.v. mas und masa (sig)). In seinem norwegischen etymologischen Wörterbuch verbindet Alf Torp genanntes Verb mit dem norwegischen Wort masast 'einschlafen, das Bewußtsein verlieren', zu dem auch das norwegische Adjektiv masen 'matt, erschöpft' gehört, und führt für diese skandinavischen Wörter die "germanische Wurzel" * mas an.

Der Schriftsteller Veijo Meri hat in seinem eigenen finnischen etymologischen Wörterbuch bereits in der ersten Auflage (1982: 129) ver- 
mutet, daß masentaa 'niederdrücken, unterdrücken, deprimieren usw.', masentua 'niedergeschlagen, verstimmt werden' sowie das Adjektiv masea 'ruhig, langsam' aus o.g. skandinavischer Wortfamilie ins Finnische übernommen wurden. Diese finnische Wortfamilie scheint damit eine brauchbare Interpretation erhalten zu haben; als gemeinsame frühe Bedeutung kann sowohl für das Skandinavische als auch für das Finnische 'kraftlos, ruhig, langsam' angenommen werden. In den finnischen Dialekten bedeuten masentua und maseta (masene-) auch 'ermüden, ermatten, ruhig, still werden' (SMSA). Martti Airila nahm an (1940: 147), daß gerade hier die ursprünglichere Bedeutung der finnischen Verben nachweisbar sei. Am Adjektiv masea, in den Dialekten und der alten Schriftsprache masia, läßt sich nachweisen, daß in der Bibel von 1642 dem Wort masia (Weisheit Salomos 16: 18) in der Übersetzung von Luther deutsch gemach entspricht. Ganander führt in seinem Wörterbuch s.v. für masia den Beispielsatz "kusa joki masein on" an, den er übersetzt mit "var ån är lugnast, stillast" (Ganander entnahm den Satz dem Almanach von 1772, s. Hormia 1962: 302).

\section{9. öykätä 'prahlen, flunkern, großtun'}

In der finnischen Gegenwartssprache begegnen neben dem Verb öykätä noch ein häufigeres Verb, die Ableitung öykkäillä mit derselben Bedeutung, sowie die Substantiva öykkääjä und öykkäri 'Angeber, Prahler, Protz, Großsprecher'. In der alten Schriftsprache treten die Wörter öykätä und öykkäri auf, die bereits Agricola oft verwendet, z.B. "Mutta ette mine Euangelium sarnan, ei minun taruitze site euckemen" (II 475, vgl. Luther: "-- darff ich mich nicht rhümen", 1. Kor. 9: 16), "Mine sanoin nijlle Euckerille, Elket nin kerskaco" (III 318, vgl. Luther: "Ich sprach zu den Rhumrettigen, Rhümet nicht so", Ps. 75: 5). Diese Wörter sind auch im Wörterbuch von Juslenius angeführt: öyckään 'jacto, glorior; skräpper, skryter', öyckäri 'jactator, thraso; storskytare'. Aus den Dialekten gibt es bis auf wenige Angaben aus einzelnen Gegenden keine weiteren Informationen über diese Wörter; belegt ist z. B. das Sprichwort "Paremp ol löytty kun öykätty" ("es ist besser etwas zu finden als aufzuschneiden') (Jämsä); dieses Sprichwort begegnet in ver- 
schiedenen parömiologischen Sammlungen aus der Zeit der schwedischen Herrschaft, die aus verschiedenen Teilen Finnlands stammen (s. Kuusi, 1953: 13); es ist auch in der 1702 gedruckten Sammlung von Florinus enthalten.

Das Verb öykätä, aus dem sich die anderen o.g. Wörter ableiten, könnte eine Entlehnung aus dem altschwedischen Verb öka (auch ökia) ‘öka, föröka, göra större' (Söderwall) sein, vgl. schwed. öka 'vermehren, vergrößern', ökas 'sich vermehren, zunehmen'. Das schwedische Verb verfügt (zumindest heute) nicht über die Bedeutung 'prahlen', jedoch konnte es auch im Finnischen gut dazu kommen, vgl. z.B. fi. suurennella 'übertreiben' (vgl. suuri 'groß'), isotella 'prahlen, großtun' (vgl. iso 'groß'), levennellä id. (vgl. leveä 'breit') usw. Der finnische Diphthong öy in der ersten Silbe ist vergleichbar mit z. B. den schwedischen Entlehnungen töytätä 'stoßen' (< schwed. stöta id.) und ( $p$ )röystäillä 'prahlen, sich mit etwas brüsten' (< schwed. brösta sig id.) (SKES).

Neben dem Verb öykätä tritt in der alten finnischen Schriftsprache auch die Form öyhkätä auf; so wären einige Formen zu lesen, die auch bei Agricola begegnen (euhkesit, auch: euchkerit). Im Wörterbuch von Renvall finden sich öyhkätä, öykätä 'prahlen, sich rühmen, stolz drohen 0 . sich betragen'. Im Wörterbuch der finnischen Gegenwartssprache (NS) ist jedoch nur öyhkiä angeführt, wonach = öhkiä = ähkiä = puhkia 'stöhnen, ächzen'. Offensichtlich entstand öyhkätä 'prahlen' zum Teil unter dem Einfluß derartiger Ausdrücke, zum Teil z.B. der Wörter pöyhkeä 'protzig, hochmütig' (Renvall: 'aufgebläht, stolz') und röyhkeä 'trotzig; frech; hochmütig' (Renvall: 'aufgebläht, stolz, prahlerisch').

\section{Literatur}

Agricola I-III = Mikael Agricolan teokset I-III. Faksimile. Helsinki-Porvoo 1931.

AHLBÄCK, OLAV 1982-: Ordbok över Finlands svenska folkmål I-. Helsingfors. 
Beiträge zu den älteren und jüngeren germanischen Lehnwörtern... 181

AIRILA, M. 1940: Johdatusta kielen teoriaan I. Porvoo-Helsinki.

ANTTILA, VEIKKO 1968: Äes Suomessa. Suomi 114:1. Helsinki.

BAETKE, WALTER 1965/68: Wörterbuch zur altnordischen Prosaliteratur

I-II. Berlin.

Bibel 1642 = Biblia, Se on coco Pyhä Raamattu, Suomexi. Stockholm, Faximile. Porvoo 1971.

BLÖNDAL, SIGFUS 1920/24: Islandsk-Dansk Ordbog. Reykjavik.

BLÖNDAL, MAGNÚSSON, ASGEIR 1989: Íslensk orððsifjabók. [S.1.]

BORDEN, ARTHUR R. JR. 1982: A comprehensive Old-English Dictionary. Lenham-New York-London.

COLLINDER, BJÖRN 1932: Die urgermanischen lehnwörter im finnischen. Skrifter utgivna av K. Humanistiska Vetenskaps-Samfundet i Uppsala 28:1. Uppsala.

DAHLGREN, F. A. 1914/16: Glossarium öfver föråldrade eller ovanliga ord och talesätt i svenska språket från och med 1500-talets andra årtionde. Fotografiskt återgivande med anslag av Svenska Akademien. Köpenhamn 1960.

DALIN, A. F. 1868: Svensk handordbok med tillägg af ordens etymologi jemte ett bihang. Stockholm.

EDW = Etymologisches Wörterbuch des Deutschen. 2. Auflage, durchgesehen und ergänzt von Wolfgang Pfeifer. Berlin 1993.

EEW = Julius Mägiste: Estnisches etymologisches Wörterbuch I-XII. Helsinki 1982-1983.

EKMS = Andrus Saareste: Eesti keele mõisteline sõnaraamat 1-4. Stockholm 1958-1968.

EURÉN, G. A. 1860: Suomalais-Ruotsalainen Sanakirja. Hämeenlinna.

FLORINUS, HENRIK 1702: Wanhain Suomalaisten Tawaliset ja Suloiset Sananlascut. Faximile. Helsinki 1987.

FREUDENTHAL, A. O. - VENDELL, H. A. 1886: Ordbok öfver estländsksvenska dialekterna. Helsingfors.

GANANDER, KRISTFRID 1786/87: Nytt Finskt Lexicon I-III. Manuskript. Faksimile. Porvoo 1937-1940.

GRÖNHOLM, MAIJA 1988: Ruotsalaiset lainasanat Turun murteessa. Åbo.

GUSTAVSON, HERBERT 1991: Gotländsk ordbok I-II. Visby.

HÄKKINEN, KAISA 1983: Suomen kielen vanhemmasta sanastosta ja sen tutkimisesta. Turku.

1990: Nykysuomen sanakirja. Etymologinen sanakirja. Toinen, tarkistettu painos. Helsinki.

HAKULINEN, LAURI 1979: Suomen kielen rakenne ja kehitys. Neljäs, korjattu ja lisätty painos. Helsinki.

HELLQUIST, ELOF 1939: Svensk etymologisk ordbok I-II. Ny omarbetad och utvidgad upplaga. Lund. 
HOFSTRA, TETTE 1985: Ostseefinnisch und Germanisch. Frühe Lehnbeziehungen im nördlichen Ostseeraum im Lichte der Forschung seit 1961. Groningen.

HOLTHAUSEN, F. 1963: Altenglisches etymologisches Wörterbuch. Heidelberg.

HORMIA, OSMO 1961: Gananderin sanakirjan lähteet. Helsinki.

HUSTAD, T. 1979: Großes Norwegisch-Deutsches Wörterbuch. Oslo-Bergen -Tromsø.

IMS = R. E. Nirvi: Inkeroismurteiden sanakirja. Helsinki 1971.

IRSSK = Iso ruotsalais-suomalainen sanakirja I-III. Helsinki 1982.

ITKONEN, TERHO 1957: Suomen kielen suksisanastoa. Helsinki.

1980: Lisiä suomen rajausoppiin. - Virittäjä 84.

JANSSON, SVEN B.F. 1989: Isländsk-svensk ordbok. Femte upplagan. Kungälv.

JOKI, AULIS J. 1973: Uralier und Indogermanen. Die älteren Berührungen zwischen den uralischen und indogermanischen Sprachen. MSFOu 151.

JUSLENIUS, DANIEL 1745: Suomalaisen Sana-Lugun Coetus. Faximile. Helsinki 1968.

KARLSSON, FRED 1983: Suomen kielen äänne- ja muotorakenne. Helsinki.

KARSTEN, T. E. 1943/44: Finnar och germaner. Folkmålsstudier IX-X. Helsingfors.

KKS = Karjalan kielen sanakirja I-III. Helsinki 1968-83.

KLUGE, FRIEDRICH - SEEBOLD, ELMAR 1989: Etymologisches Wörterbuch der deutschen Sprache. 22. Auflage unter Mithilfe von Max Bürgisser und Bernd Gregor völlig neu bearbeitet. Berlin-New York.

KOIVULEHTO, JORMA 1981: Zur Periodisierung germanischer Lehnwörter im Ostseefinnischen. Vortrag gehalten an der Rijksuniversiteit Groningen am 12.5.1981. Vervielfältigung.

KUUSI, MATTI 1953: Vanhan kansan sananlaskuviisaus. Helsinki.

LEHMANN, WINFRED P. 1986: A Gothic Etymological Dictionary. Leiden.

LEHTINEN, TAPANI 1979: Itämerensuomen verbien historiallista johtooppia. Suomen avajaa, karkajaa -tyyppiset verbit ja niiden vastineet lähisukukielissä. MSFOu 169.

LESKINEN, HEIKKI 1992: Karjalan kielikartasto I. Jyväskylän yliopiston Suomen kielen laitoksen julkaisuja 33. Jyväskylä.

LINDÉN, EEVA 1942: Kaakkois-Hämeen murteiden äännehistoria I. Konsonantit. Helsinki.

LÖNNROT, ELIAS 1874/80, 1886: Suomalais-Ruotsalainen Sanakirja I-II. Lisävihko Elias Lönnrotin Suomalais-Ruotsalaiseen sanakirjaan. Toimittanut A. H. Kałlio. Kolmas, manual-menetelmällä jäljennetty painos. Porvoo-Helsinki 1958.

LÜHR, ROSEMARIE 1988: Expressivität und Lautgesetz im Germanischen. Heidelberg. 
LUTHER, D. MARTIN 1544/45: Die gantze Heilige Schrift Deudsch. Wittenberg. Letzte zu Luthers Lebzeiten erschienene Ausgabe. Herausgegeben von H. Volz unter Mitarbeit von H. Blanke. Textredaktion F. Kur. München 1972.

MERI, VEIJO 1982: Sanojen synty. Etymologinen sanakirja. Jyväskylä.

MIKKONEN, PIRJO - PAIKKALA, SIRKKA 1992: Sukunimet. Toinen painos. Helsinki.

MSzFE = A magyar szókészlet finnugor elemei. Etimológiai szótar I-IV. Budapest 1967-81.

NIELSEN, NIELS ÅGE 1976: Dansk etymologisk Ordbog. 3. reviderede udgave med et tillæg. København.

NS = Nykysuomen sanakirja I-VI. Porvoo 1951-1961.

$\mathrm{OD}=$ The Concise Oxford Dictionary of Current English. Oxford 1951.

$\mathrm{ODEE}=\mathrm{C}$. T. Onions (ed.): The Oxford Etymology of English. Reprinted with corrections. Oxford 1969.

ÖSTERGREN, OLOF 1919/72: Nysvensk ordbok I-X. Stockholm.

POKORNY, JULIUS 1959: Indogermanisches etymologisches Wörterbuch I-II. Bern-München.

RAPOLA, MARTTI 1966: Suomen kielen äännehistorian luennot. Helsinki. RAUN, ALO 1982: Eesti keele etümoloogiline teatmik. Rooma-Toronto. RENVALL, GUSTAVUS 1826: Suomalainen Sana-Kirja. Åbo.

SAMMALLAHTI, PEKKA 1988: Historical Phonology of Uralic Languages with Special Reference to Samoyed, Ugric, and Permic. - In: Denis Sinor (ed.): The Uralic Languages. Description, History and Foreign Influences. Leiden-New York-København-Köln.

Sanastaja. Aikakauslehti sanatietoja ja -tiedusteluja varten. Kotimaisten kielten tutkimuskeskus. Helsinki.

SKES = Suomen kielen etymologinen sanakirja I-VI. Helsinki 1955-1978. SMS = Suomen murteiden sanakirja I-III. Helsinki 1985-1993.

SMSA = Suomen murteiden sana-arkisto. Kotimaisten kielten tutkimuskeskus. (Finnisches Dialektarchiv. Forschungszentrum für die Landessprachen Finnlands.) Helsinki.

SÖDERWALL, K. F. 1884, 1918, 1925/73: Ordbok öfver svenska medeltidsspråket I-II. Supplement av K. F. Söderwall, W. Åkerlund, K. G. Ljunggren, E. Wessén. Lund.

SSA = Suomen sanojen alkuperä. Etymologinen sanakirja I. Helsinki 1992. STREITBERG, WILHELM 1919/28: Die gotische Bibel I-II. Heidelberg.

STRENG, H. J. 1915: Nuoremmat ruotsalaiset lainasanat vanhemmassa suomen kirjakielessä. Helsinki.

SVJ = М. И. Зайцева - М. И. Муллонен: Словарь вепсского языка. Ленинград 1972.

TALVE, ILMAR 1979: Suomen kansankulttuuri. Helsinki. 
TORP, ALF 1919: Nynorsk etymologisk ordbok. Kristiania.

TUOMI, TUOMO 1990: Järviseudun murteesta. In: Järviseudun historia III. Kokkola.

TUOVINEN, INKERI 1931: Tornionjoen takaa suomea oppimassa. - Virittäjä 35.

UEW = Károly Rédei: Uralisches etymologisches Wörterbuch I-II. Budapest.

VENDELL, H.A. 1884: Samlingar af ord ur Nyländska allmogemålet. Helsingfors.

VENDELL, HERMAN 1904/06: Ordbok over de östsvenska dialekterna 1-4. Helsingfors.

VILKUNA, KUSTAA 1970: Sanoja niityltä niitetyltä. - Virittäjä 74.

Virittäjä. Kotikielen Seuran aikakauslehti. Helsinki.

VIRTARANTA, PERTTI 1946, 1957: Länsiylăsatakuntalaisten murteiden äännehistoria I. Konsonantit. II. Vokaalit. Helsinki.

VKKMS = Lauri Posti - Seppo Suhonen: Vatjan kielen Kukkosin murteen sanakirja. Helsinki 1980.

VKS = Vanhan kirjasuomen sanakirja I. Helsinki 1985.

VMS = Väike murdesõnastik I-II. Toimetanud V. Pall. Tallinn 1982, 1989.

VRIES, JAN DE 1962: Altnordisches etymologisches Wörterbuch. 2. verbesserte Auflage. Leiden.

1971: Nederlands etymologisch woordenboek. Leiden.

VUORELA, TOIVO 1979: Kansanperinteen sanakirja. Helsinki.

WAHRIG, GERHARD 1986: Deutsches Wörterbuch mit einem "Lexikon der deutschen Sprachlehre". Neuausgabe. München.

WESSMANN, V. E. V. 1925/32: Samling av ord ur östsvenska folkmål I-II. Helsingfors.

WIEDEMANN, F. 1893: Ehstnisch-deutsches Wörterbuch. Zweite vermehrte Auflage. St. Petersburg.

WIIK, KALEVI 1969: Onko suomen yleiskielessä oppositiota /i/ - /j/ ja / $/$ / /v/? - In: Juhlakirja Paavo Siron täyttäessä 60 vuotta. Acta Universitatis Tamperensis A: 26. Tampere. 